\title{
ANII
}

\section{ASSOCIAÇÃO DO TABAGISMO COM A TUBERCULOSE E SEUS DESFECHOS NEGATIVOS: UMA REVISÃO SISTEMÁTICA}

\author{
Alessandro Rolim Scholze ${ }^{1}$, Laura Terenciani Campoy², Marcos Augusto Moraes Arcoverde ${ }^{3}$, \\ Josilene Dália Alves ${ }^{4}$, Miguel Fuentealba-Torres ${ }^{5}$, Ricardo Alexandre Arcêncio ${ }^{6}$.
}

RESUMO

Objetivo: Identificar as evidências de associação entre a tuberculose e o tabagismo, bem como as complicações do tabaco para o paciente com tuberculose.

Método: Trata-se de revisão sistemática da literatura, estruturada pelas etapas do PRISMA. Utilizou-se a estratégia PEO (population, exposure e outcome) para elaboração da pergunta norteadora: "O tabaco está associado a tuberculose?" e "Quais as complicações decorrentes do tabagismo no tratamento da tuberculose?". Para a busca dos artigos, definiu-se um limite temporal de 1999 a 2019, sendo efetuada nas bases de dados PubMed, Cumulative Index to Nursing and Allied Health Literature e Literatura Latino-americana e do Caribe em Ciências da Saúde. Utilizaram-se os descritores "Tabaco", "Consumo de tabaco", "Tuberculose", "Adulto", isolados e/ou combinados. Foram incluídos artigos disponíveis na íntegra nos idiomas português, inglês e espanhol. O software State of the Art through Systematic Review foi utilizado para o gerenciamento do banco de dados.

Resultados: Foram recuperados 227 artigos. Destes, 25 foram incluídos e examinados na íntegra, sendo que apenas 3 foram desenvolvidos a nível nacional. Os resultados apontaram que existe uma associação entre o tabagismo e o desenvolvimento da tuberculose. Entre as complicações no tratamento, encontram-se falha no tratamento, não adesão e abandono, sendo a mortalidade maior entre os pacientes que eram tabagistas. Também evidenciou que a cura da tuberculose estava associada ao paciente que nunca fumou.

Conclusão: $O$ conjunto de estudos revisados indica que, além do tabagismo apresentar forte associação com a tuberculose, o uso do tabaco aumenta as chances de desfechos desfavoráveis ao tratamento da tuberculose.

Descritores: Tabaco; Uso de tabaco; Tuberculose.

1. Alessandro Rolim Scholze - Enfermeiro. Mestre. Universidade Estadual do Norte do Paraná. Professor colaborador. Bandeirantes, Paraná, Brasil. E-mail: scholze@uenp.edu.br. ORCID iD: https://orcid.org/0000-0003-4045-3584.

2. Laura Terenciani Campoy - Enfermeira. Mestre. Escola de Enfermagem de Ribeirão Preto, Universidade de São Paulo/EERP-USP. Ribeirão Preto, São Paulo, Brasil. E-mail: lauratcampoy@hotmail.com. ORCID iD: https://orcid.org/0000-0002-9338-9689

3. Marcos Augusto Moraes Arcoverde - Enfermeiro. Doutor. Universidade Estadual do Oeste do Paraná, Foz do Iguaçu, Paraná, Brasil. E-mail: marcos.arcoverde2013@gmail.com. ORCID iD: https://orcid.org/0000-0001-5104-559X

4. Josilene Dália Alves - Enfermeira. Doutora. Escola de Enfermagem de Ribeirão Preto, Universidade de São Paulo/EERP-USP. Ribeirão Preto, São Paulo, Brasil. E-mail: josydalia@hotmail.com. ORCID iD: https://orcid.org/0000-0001-5007-9536

5. Miguel Fuentealba-Torres - Enfermeiro. Doutor. Escola de Enfermagem de Ribeirão Preto, Universidade de São Paulo/EERP-USP. Ribeirão Preto, São Paulo, Brasil. E-mail: elmagallanico@gmail.com. ORCID iD: https://orcid.org/0000-0003-4343-6341.

6. Ricardo Alexandre Arcêncio - Enfermeiro. PHD. Escola de Enfermagem de Ribeirão Preto, Universidade de São Paulo/USP. Ribeirão Preto, São Paulo, Brasil. E-mail: ricardo@eerp.usp.br ORCID iD: http://org/0000-0003-4792-8714.

\section{Autor Correspondente}

Alessandro Rolim Scholze

Endereço: Rua Prefeito José Mario Junqueira, n. 393 ap.01.

Bandeirantes, PR, Brasil.

E-mail: scholze@uenp.edu.br. Telefone: (43) 99602-8846

Data de submissão: 18/06/2019

Data de aceite: $13 / 09 / 2019$

Seção a que o manuscrito se destina: Artigo de revisão.

Como citar esse artigo

SCHOLZE, A. R, et al. Associação do tabagismo com a tuberculose e seus desfechos negativos: uma revisão sistemática. Advances in Nursing and Health, v. 1, p. 113-126, Londrina, 2019. 


\section{INTRODUÇÃO}

A tuberculose (TB) está entre as dez principais causas de morte dentre as doenças infecciosas no mundo, com 10,4 milhões de registros em 2016, sendo que 1,8 milhão evoluíram ao óbito. Contudo, a Organização Mundial de Saúde (OMS) lançou a estratégia "End TB" para o período pós 2015, que tem como meta a eliminação da doença ( $<1$ caso por 100.000 habitantes) e ainda promover a redução da mortalidade por TB em 95\% até o ano de 2035[1].

Assim, o combate à TB enfrenta muitos desafios quanto à organização dos serviços de saúde, às políticas públicas para o enfrentamento da doença, bem como aos fatores socioeconômicos, clínicos e comportamentais. Dentre esses, destaca-se a associação da TB às outras comorbidades, como o tabagismo, que pode dificultar o tratamento, aumentar as chances de transmissão e contribuir para um aumento da morbimortalidade dos indivíduos[2,3].

Estima-se que 1,3 bilhão de pessoas no mundo consomem tabaco, sendo que a maioria desses indivíduos vive em países subdesenvolvidos ou em desenvolvimento, onde as taxas de TB são mais elevadas[4,5].
Nesse contexto, atualmente, evidencia-se uma epidemia tabágica crescente, sendo o controle da TB ainda precário. Contudo, as perspectivas futuras são preocupantes em relação à associação de ambos os agravos. Estudo que avaliou o impacto do tabagismo na incidência de TB, baseado na curva de tendência do tabagismo e na projeção de incidência, prevalência e mortalidade de TB entre o período de 2010 a 2050, estimou que o tabagismo produzirá um excesso de 18 milhões de casos de TB e de 40 miIhões de mortes, caso o número de fumantes no mundo continue com a mesma tendência, dificultando ainda mais a possibilidade de se atingir as metas estipuladas pela OMS[6].

No Brasil, onde o tabagismo ainda é preocupante, em 2013 havia 15\% de fumantes entre adultos e uma prevalência maior entre brasileiros com menor nível de escolaridade[7]. De acordo com um estudo de coorte, os fumantes apresentam risco 2,5 vezes maior de desenvolver TB recorrente, comparados com os não-fumantes, além de mostrarem pior adesão ao tratamento da TB[8].

Apesar dos níveis de evidências da associação entre tabagismo e TB, as principais diretrizes mundiais sobre TB trazem pouca informação sobre a necessidade do comba- 
te ao tabagismo para melhorar o controle da TB[9], necessitando assim de mais estudos e evidências que comprovem tal associação e suas possíveis causas. Diante do exposto, o objetivo deste estudo foi identificar as evidências de associação entre a tuberculose e o tabagismo, bem como as complicações do tabaco para o paciente com tuberculose.

\section{MÉTODO}

Para identificar as evidências de associação entre o tabaco e a TB perante as complicações do tratamento em pacientes com diagnóstico de TB, foi conduzida uma revisão sistemática da literatura, segundo as etapas do PRISMA: 1) Identificação; 2) Triagem; 3) Elegibilidade, 4) Inclusão[10]. Na primeira etapa (identificação), foram desenvolvidas estratégias de busca a partir de duas questões de pesquisa estruturadas segundo os componentes 1) População, 2) Exposição, 3) Outcome (PEO) (P - Population - Pessoas adultas, E - exposure - Tabaco, O - Outcome - Tuberculose).

Assim, para atingir o objetivo da revisão, foram formuladas as perguntas: 1) 0 tabaco está associado à tuberculose?; 2)
Quais as complicações decorrentes do tabagismo no tratamento da tuberculose?. Para efetuar a estratégia de busca, foram recuperados estudos publicados entre o período de 01 de janeiro de 1999 e 31 de maio de 2019.

As buscas foram desenvolvidas em articulação com as questões de pesquisa, sendo utilizadas as seguintes bases de dados: PubMed, Cumulative Index to Nursing and Allied Health Literature (CINAHL) e Literatura Latino-americana e do Caribe em Ciências da Saúde (LILACS). Foram utilizados os descritores Mesh Terms para as bases de dados internacionais, ((Tuberculosis) AND (Smoking) OR (Tobacco) AND (adult)) e Descritores em Ciências da Saúde (Decs) para as bases de dados nacionais ((Adultos) AND (Tabaco) AND (Tuberculose)) e os operadores booleanos AND e OR para as combinações entre os descritores.

Na etapa de triagem, a qual se caracterizou pela leitura dos títulos, resumos e palavras-chave dos 217 artigos selecionados, foram adotados os seguintes critérios de elegibilidade: pessoas adultas com idade superior a 18 anos, consumo ativo de tabaco em algum momento da vida e que tivessem o diagnóstico/associação com a TB. 
Para a leitura desses artigos, utilizou-se o software State of the Art through Systematic Review (StArt), que tem como objetivo realizar o gerenciamento do banco de dados.

Assim, após a eliminação das duplicidades, os artigos foram submetidos à triagem. Os estudos potencialmente elegíveis foram pré-selecionados a partir da leitura dos títulos, resumos e palavras-chave, sendo efetuada por dois revisores independentes (ARS - MF). A leitura independente consiste em uma análise individualizada que só após a conclusão da triagem comparará os artigos "incluídos ou excluídos". Caso ocorra uma discrepância entre os revisores, o artigo passa por uma terceira análise (MAMA).

A etapa de elegibilidade consiste na leitura na íntegra dos estudos potencialmente elegíveis, sendo efetuada por cinco revisores (ARS - MF - LC - JA - MAMA).

Já na etapa de inclusão os avaliadores realizam a extração dos dados, a partir do protocolo pré-definido. Esse protocolo tem como objetivo facilitar e nortear o processo de extração de informação dos artigos durante a leitura na íntegra. Ao final, as referências são exportadas para um banco de dados em Excel para construção e análise dos resultados.

\section{RESULTADO}

Recuperou-se um total de 227 artigos, sendo que 110 foram identificados na base de dados Pubmed, 64 na CINAHL e 53 na LILACS. Após remoção de duplicados e aplicação dos critérios de inclusão, selecionaram-se 74 artigos para leitura do texto na íntegra. Por fim, 25 estudos foram incluídos e examinados pelos autores. Os processos de busca e seleção são apresentados na Figura 1, utilizando o fluxograma do PRISMA[10].

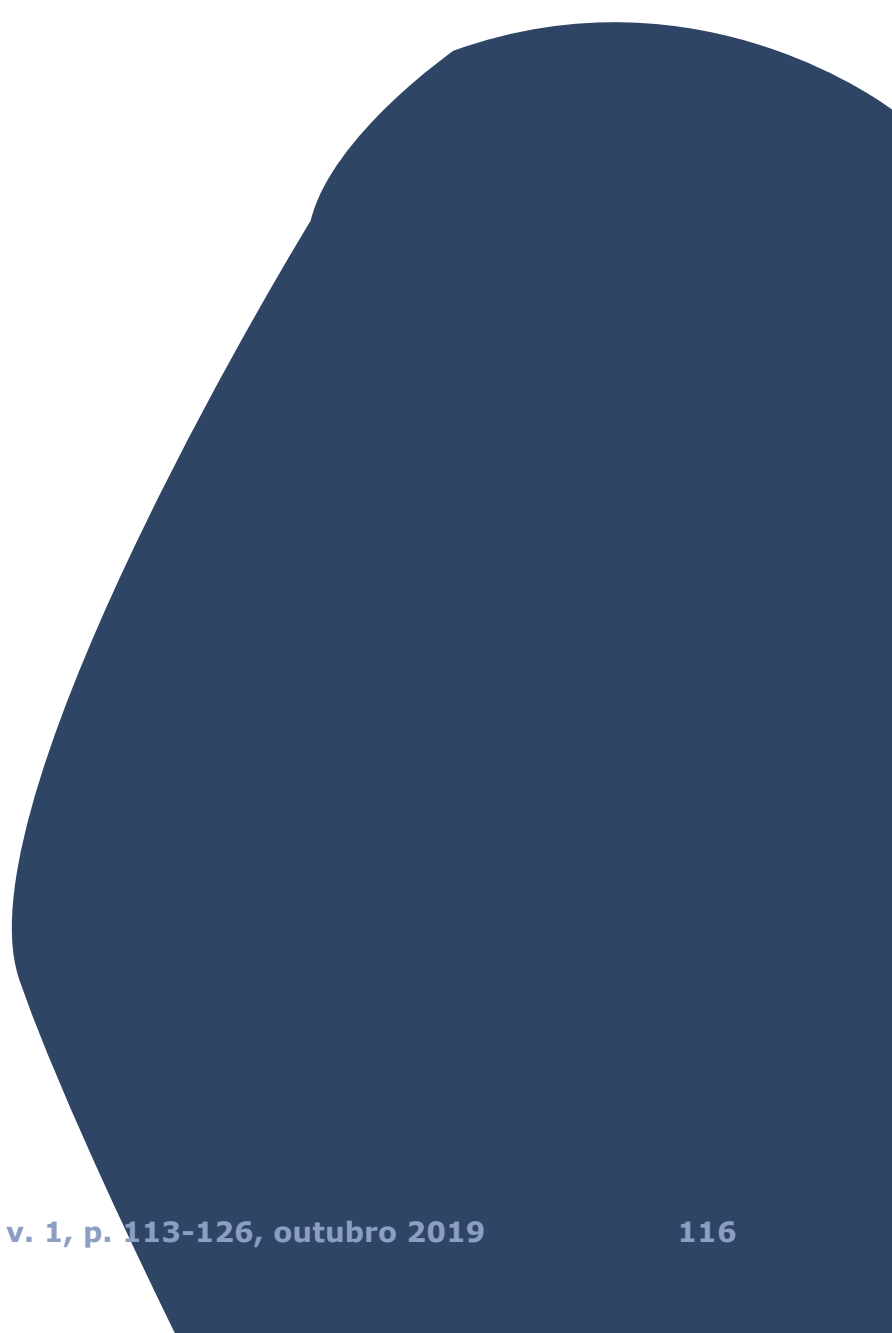


Artigos identificados em bases de dados PUBMED $(n=110) \mathrm{Cl}-$ NAHL [ $n=64]$ LILACS $(n=53)$ Total $(n=227)$

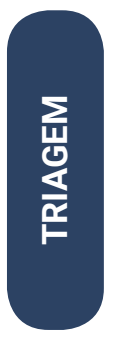

Artigos restantes após remoção de duplicados $(n=217)$

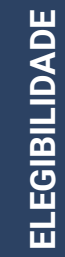

Artigos selecionados $(n=217)$ Artigos excluídos ( $n=143$ )
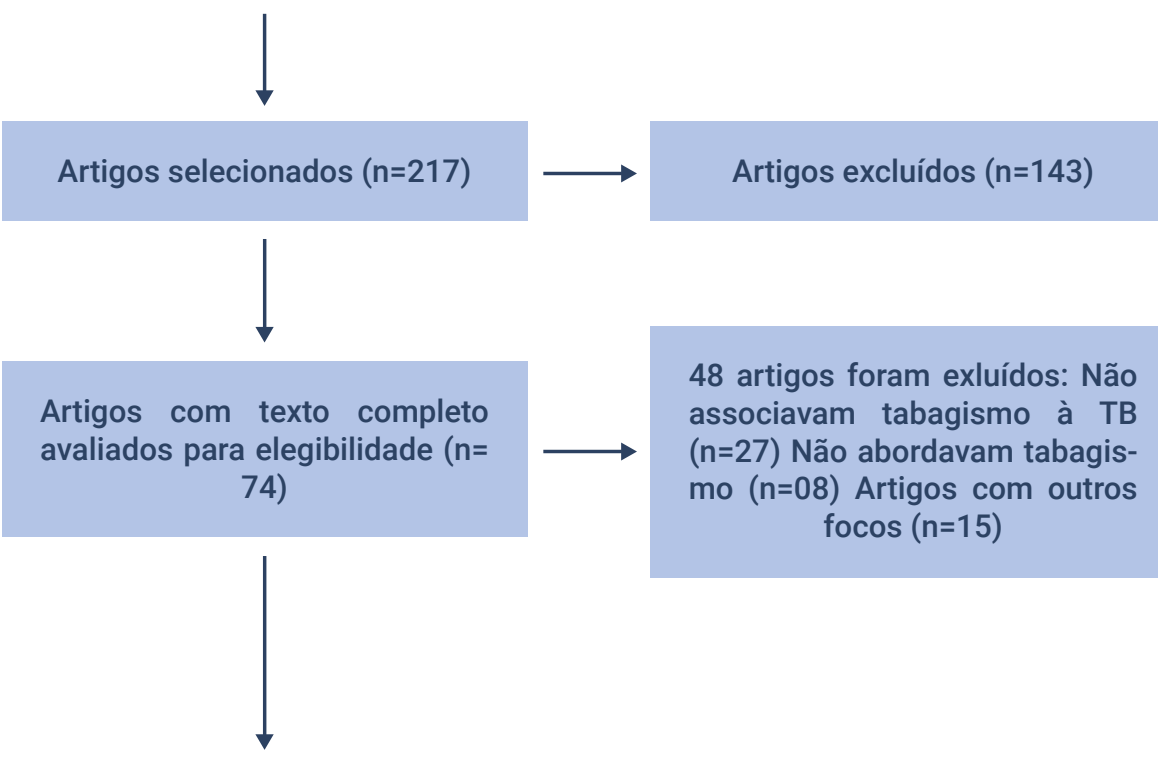

Estudos incluídos na revisão $(n=25)$

Figura 1 - Fluxograma dos estudos incluídos na revisão sistemática.

Os dados essenciais extraídos dos artigos incluídos estão resumidos no

Quadro 2. Os resultados da revisão da literatura apontaram que existe uma importante associação entre o tabagismo e o desenvolvimento da TB.
Os resultados mostraram que seis estudos abordaram problemas no tratamento (faIha, não adesão e abandono) como uma das principais complicações do tabagismo para os pacientes com TB (ID - 3,9,16,22,23,25). Um estudo mencionou que o tabagismo 
influencia negativamente na concentra-

ção da pirazinamida, droga utilizada no

tratamento da TB (ID-13).

Além do tabaco, outras drogas também estiveram relacionadas com a TB e suas com-

plicações, entre elas o álcool, que foi mencionado em seis estudos (ID - 1,10,6,18,20,22).

Quanto à mortalidade, três estu-

dos detectaram que a mortalidade por

TB foi maior entre pacientes que eram

tabagistas (ID - 9,10,24).

\section{DISCUSSÃO}

Esta revisão tem como objetivo identificar evidências científicas de associação entre o consumo de tabaco, seja passiva ou ativamente, e a TB, mediante achados sobre as complicações e o desfecho do tratamento de TB entre tabagistas. Foi possível identificar que houve um total de 25 artigos, sendo que a maioria, 22 artigos, eram estudos internacionais, com os cenários localizados no continente asiático.
1 Balakrishnan

2019 Índia

Higher risk of developing active TB among adult diabetics exposed to TB during childhood: A study from Kerala, India

2 Nandasena S. et al.(12) 2019 Sri Lanka

Characteristics and sputum conversion of tuberculosis (TB) patients in Kalutara, Sri Lanka

3 Oliveira SM. et al.(13) 2018 Brasil

Predictors of noncompliance to pulmonary tuberculosis treatment: An insight from South America

4 Tahseen S. et al.(14) 2018 Paquis-

Systematic case finding for tuberculosis in HIV-infected people who inject drugs: experience from Pakistan

Prevalence and patterns of cigarette smoking among patients co-infected with human immunodeficiency virus and tuberculosis in Tanzania

The Association between Active and Passive Smoking and Latent Tuberculosis Infection in Adults and Children in the United States: Results from NHANES
Identificar os fatores de risco em nível individual para a TB.

Descrever as características dos pacientes com TB e avaliar os fatores associados à conversão do escarro.

Investigar os fatores associados ao maior risco de abandono do tratamento da TB em Porto Alegre, Brasil.

Avaliar a prevalência de TB entre usuários de drogas injetáveis infectadas pelo HIV encaminhados para o Tratamento Antirretroviral e avaliar o valor diagnóstico da tosse como um sintoma de rastreamento.

Determinar o padrão e a prevalência do tabagismo entre pacientes coinfectados com HIV e TB em Dar es Salaam, Tanzânia.

Determinar a associação entre tabagismo ativo e passivo na infecção por TB latente em uma amostra representativa de adultos e crianças nos EUA.
Contato próximo durante a infância e recente com TB, fumar mais de 10 cigarros por dia e consumir mais de 10 doses de bebida alcoólica por semana foram considerados um fator associado para a TB.

Quem fuma tabaco é menos propenso a ter conversão de escarro em 2-3 meses em comparação com os não-fumantes. A abstinência do fumo e do consumo de álcool são importantes fatores para a conversão do escarro em pacientes com TB pulmonar.

Fatores associados à não adesão ao tratamento foram $72 \%$ maiores em indivíduos que fumam e $173 \%$ maiores entre os indivíduos que usaram drogas. Viver em uma área de baixa renda, com abuso de drogas e história de tabagismo permaneceram associados ao abandono do tratamento.

Uma maior prevalência de TB ocorreu entre fumantes com uma história de mais de 10 cigarros/dia do que naqueles com menor de 10 cigarros/dia.

Homens que eram tabagistas e consumiam álcool apresentaram sete vezes mais chances de desenvolver TB.

Fumantes ativos eram significativamente mais propensos a ter infecção latente por TB do que adultos não-fumantes. Os fumantes passivos adultos também apresentaram maior chance de infecção latente por TB em comparação aos não-fumantes. 
8 Alavi-Naini R. et al.(17)

2012

Irã

Association Between Tuberculosis and Smoking
Determinar a associação entre

TB e tabagismo no sudeste do Irã, uma área endêmica para tuberculose.

Fumantes de cigarros foram 3,1 vezes mais frequentes em pacientes com TB em comparação com não-fumantes. Outros fatores que evidenciaram diferenças significativas entre pacientes com TB foi o uso de medicamentos não parenterais, história familiar de TB, história familiar de tabagismo e história de tabagismo superior a 10 anos.

Identificar o impacto do tabagisClinical Modalities and Therapeutic Outcomes; Between Ever-Smokers Versus Never-Smokers of Tuberculosis Patients in Penang, Malaysia

(18) et 2010 Malásia et al.(19) 2008 Brasil

Perfil de pacientes que evoluem para óbito por tuberculose no município de São Paulo, 2002 al.(20)

Does smoking explain sex differences in the global tuberculosis epidemic?

$\mathrm{H}$ e mant h al.(22)

Diagnosing active and latent tuberculosis among Iranian HIV-infected patients

mortality from TB and other diseases in India: retrospective study of 43000 adult male deaths and 35000 controls

Pharmacokinetics of thrice-weekly rifampicin, isoniazid and pyrazinamide in adult tuberculosis patients in India

eterminants of active pulmonary tuberculosis in Ambo Hospital, West Ethiopia

mo nas características clínicas

e no desfecho do tratamento

da $T B$, e enfatizar que essa associação pode ser útil no tratamento de casos de TB.

Descrever o perfil de pacientes adultos residentes no município de São Paulo que evoluíram para óbito associado à TB, segundo fatores biológicos, ambientais e institucionais.

Determinar se o tabagismo faz uma contribuição significativa para as diferenças sexuais na distribuição da TB entre países de alta carga.

Avaliar a mortalidade específica por idade do tabagismo entre os homens (uma vez que poucas mulheres fumam) na Índia urbana e rural.

Estudar a farmacocinética de rifampicina, isoniazida e pirazinamida em pacientes adultos com tuberculose e examinar fatores que influenciam a farmacocinética da droga.

Rastrear TB entre pacientes HIV positivos através de história e sintomatologia, exame físico, radiografia de tórax, tuberculina teste, assim como esfregaço de escarro e cultura para TB.

Determinar os fatores associados à TB pulmonar ativa observada nos casos do Hospital de Ambo, na Etiópia.

Tobacco smoking and tuberculosis Avaliar o efeito do tabagismo treatment outcomes: a prospective sobre o resultado do tratamento cohort study in Georgia

Tuberculosis risk factors among tuberculosis patients in Kampala Uganda: implications for tuberculosis control

al.(26)
al al.(27)
2014
Irlanda
Survival analysis of adult tuberculosis disease

\section{da TB em Tbilisi, Geórgia.}

Determinar a prevalência de diferentes fatores de risco de tuberculose e apresentação de TB entre pacientes com tuberculose em Kampala Uganda.

Estimar o tempo de sobrevida, incluindo o tempo mediano de sobrevida, e avaliar a associação e o impacto de covariáveis (fatores de risco de TB) para o evento.
A maioria dos pacientes curados nunca fumou enquanto aqueles que abandonaram o tratamento ou faleceram eram fumantes. Houve um alto risco de morte em pacientes com TB que eram tabagistas.

Entre os pacientes que evoluíram ao óbito, $71 \%$ eram tabagistas e $64 \%$ dependentes de álcool.

0 consumo de cigarros foi um preditor significativo para as notificações de TB. 0 tabagismo é um importante fator modificável que tem um impacto significativo na epidemiologia global da TB, enfatizando a importância do controle do tabagismo em países com alta incidência de TB.

0 tabagismo aumenta a incidência de tuberculose clínica, sendo a causa de metade das mortes masculinas por TB e de um quarto de todas as mortes masculinas na meia-idade.

0 tabagismo foi um fator que influenciou negativamente na concentração de pirazinamida, droga de tratamento da TB.

0 tabagismo permanece associado ao desenvolvimento de TB.

Ter um ou mais membros da família com histórico de TB, estado civil, sexo masculino, residência em área rural, ser um fumante atual ou no passado, IMC < 18,5 , infecção pelo HIV e história de infestação por vermes permaneceram como fatores significativos relacionados à TB ativa.

Fumantes ativos tiveram um risco aumentado de resultado ruim do tratamento. Aqueles que pararam de fumar há mais de dois meses antes do início do tratamento não tiveram risco aumentado.

A prevalência dos fatores de risco para TB foram HIV, diabetes, contato próximo, antecedentes familiares, tabagismo, pobreza, superlotação e uso de álcool.

0 risco para TB foi uso indevido de drogas $(2,2$ vezes), tabagismo ( 1,8 vezes), álcool ( 1,8 vezes) e medicação imunossupressora ( 3,0 vezes). 
Estimar a associação entre o Diabetes mellitus, smoking status, DM e o tempo de conversão da and rate of sputum culture conver- cultura de escarro em pacientes
19

Magee MJ. et 2014 Georgia sistant tuberculosis: a cohort study

from the country of Georgia

Predictors of TB and antiretroviral Naidoo P. et 2013 África do al.(29)

medication non-adherence in public primary care patients in South Africa: a cross sectional study al.(30) 2013 Camboja

Cigarette smoking and tuberculosis in Cambodia: findings from a national sample

Factors associated with treatment default by tuberculosis patients in Fez, Morocco K. et 2013 Marro al.(31)

$\cos$

Outcomes of tuberculosis treatment: a retrospective cohort analysis of smoking versus non-smoking patients in Penang, Malaysia

Prospective study of smoking and tuberculosis in India

Risk factors for default from TB treatment in HIV-infected individuals in the state of Pernambuco, Brazil: a prospective cohort study

Maruza M. et $2011 \quad$ Brasil
Examinar a associação entre tabagismo (principalmente fumar cigarro) e tuberculose em Mumbai, Índia.

adultos com TB pulmonar. Secundariamente, determinar fatores associados às taxas de conversão de cultura de escarro.

Investigar os fatores associados à adesão aos medicamentos anti-TB e HIV.

Examinar a relação entre tabagismo diário e história de tuberculose ao longo da vida em uma amostra nacional de adultos no Camboja.

Identificar os determinantes do padrão de tratamento da TB em Fez, Marrocos.

Determinar a prevalência de tabagismo entre os pacientes com TB em Penang e comparar os resultados do tratamento entre os pacientes com e sem tabagismo.

A taxa de conversão de cultura de escarro foi significativamente menor em pacientes tabagistas ativos, baixo índice de massa corporal, resistência a medicamentos de segunda linha, com cavidades pulmonares e com TB disseminada.

Preditores de não-adesão comuns aos medicamentos anti-TB e à terapia dupla (antirretroviral $e$ medicamentos anti-TB) incluíram a pobreza, um ou mais estados de saúde comórbidos, uso de álcool, ter parceiro HIV positivo e o uso do tabaco.

Houve uma relação positiva significativa entre TB e número de cigarros fumados por dia e maços por ano.

Os fatores preditivos para o padrão de tratamento foram: caso de recidiva, tabagismo atual, uso de álcool, estar há mais de $\mathbf{3 0}$ minutos do centro de saúde e percepção de ter recebido explicação insuficiente sobre a doença.

Pacientes com TB e fumantes tinham cerca de sete vezes mais probabilidade de falhar no tratamento ou interromper o tratamento, sendo menos propensos a serem curados de TB em comparação com os que nunca fumaram.

0 risco de mortalidade por TB entre os fumantes foi 2,6 vezes maior do que entre os não-fumantes, com uma tendência significativa para a frequência diária de cigarros fumados. Além disso, a prevalência de TB autorrelatada entre os fumantes foi de 5,2 vezes maior do que entre os não-fumantes.

Identificar os fatores de risco para o padrão de tratamento da TB em pessoas que vivem com o HIV.
As variáveis associadas ao abandono do tratamento de TB foram: sexo, idade, tabagismo, nível de escolaridade, contagem de células T CD4 e terapia altamente eficaz com antirretrovirais.

\section{Quadro 2 - Características gerais dos estudos incluídos na revisão de literatura.}

Ao analisar os artigos desta revisão, nota-se uma lacuna de conhecimento referente a publicações/desenvolvimento de pesquisas que relacionem o tabaco com TB no Brasil, com apenas três estudos desenvolvidos a nível nacional. Destes, dois associaram que a prática do tabagismo no passado ou tabagista atual é um comportamento que aumenta as chances para o abandono no tratamento, ao comparar com uma população com
TB que não possuía o hábito ou histórico de tabagismo[13-34].

\section{O terceiro estudo evidenciou que cerca} de $71 \%$ dos pacientes que tinham TB e eram tabagista evoluíram a óbito, sendo que a dependência do álcool estava presente em 64\% dos óbitos[19].

Cabe salientar que o tabagismo é um dos principais fatores de risco para mais de 
20 tipos de doenças, dentre elas a doença pulmonar obstrutiva crônica, a doença cardiovascular, o acidente vascular encefálico e os cânceres múltiplos/pulmão[35].

A relação do tabaco com a TB ocorre porque a nicotina é um fator de risco para o declínio das funções pulmonares. Constata-se que fumar desencadeia uma maior vulnerabilidade para a redução do volume expiratório, além da fumaça gerada pelo cigarro provocar danos ao organismo, a qual afeta o parênquima pulmonar e as artérias pulmonares, causando a obstrução irreversível dos ramos pulmonares[36]. Portanto, o tabaco irá causar danos histopatológicos no pulmão, afetando o sistema imune, o que o torna suscetível para infecções pulmonares, entre elas a TB[17].

Nesse contexto, a exposição passiva/ ativa ou histórico atual/pregresso de consumo do tabaco associa-se diretamente a um pior prognóstico no tratamento da TB[37]. Assim, visualiza-se um grave problema de saúde pública nos países onde existe uma alta prevalência de tabagistas. Ao existir uma população que desencadeia um alto padrão no consumo de tabaco, tal hábito aumenta os riscos para a persistência ou o surgimento de novos casos de TB, além de diminuir os índices de cura.
Fica evidente que há uma associação entre TB e tabagismo, em vários estudos[12,14-18,20-27,30-32]. Entretanto, esta revisão avança no conhecimento por apontar de qual forma essa associação interfere o prognóstico do tratamento da TB, ou seja, a quais desfechos desfavoráveis do tratamento da TB o uso abusivo de tabaco estaria associado[11-14,18,20,22,28,31].

Nesse sentido, um desfecho desfavorável identificado nesta revisão foi o abandono do tratamento. Assim, considera-se abandono do tratamento quando o paciente permanece cerca de 30 dias consecutivos sem comparecer à unidade de saúde após a data de aprazamento do retorno[38]. Além disso, ressalta-se que o esquema terapêutico para o tratamento da TB a nível nacional é gratuito e desenvolvido na atenção básica, sendo altamente efetivo, com um índice de cura satisfatóri[39], desde que o paciente siga o esquema terapêutico corretamente e sem interrupções.

Dados evidenciam que taxa de abandono do tratamento da TB no Brasil é de aproximadamente $17 \%$. Nesse sentido, esse abandono causa inúmeras consequências negativas para o paciente e para o sistema de saúde pública, visto que, ao ter um aban- 
dono, também ocorre a necessidade de um reingresso do esquema terapêutico, fator que provoca uma dificuldade na diminuição do número de novos casos da TB e o surgimento da TB-multirresistência[40].

Nessa perspectiva, quatro estu$\operatorname{dos}[13,25,32,34]$ indicaram que os fumantes tinham cerca de sete vezes mais probabilidade de falha no tratamento ou de interromper o tratamento, sendo menos propensos a serem curados de TB[34]. Evidenciou que entre os pacientes que nunca fumaram ou cessaram o tabaco há no mínimo dois meses antes de iniciar o esquema terapêutico houve mais chances de evoluir a cura ao comparar com os tabagistas[25]. Outros fatores também se associaram ao abandono do tratamento, como fazer uso de álcool, estar há mais de 30 minutos do centro de saúde, receber explicação insuficiente sobre a doença pelos profissionais da saúde[13,31].

A literatura menciona a existência de consequências negativas ocasionadas pelo tabaco na população geral. Estima-se que mais de 7 milhões de pessoas morrem por ano por doenças relacionadas ao consumo de tabaco, sendo previsto que até 2030 esse número aumente para 8,3 milhões, principalmente em países de renda baixa e média[41].
Vale constatar também que o tabaco é uma das principais causas de morte evitável em todo o mundo.

Nesse cenário, pacientes com diagnóstico de TB que são tabagistas encontram-se com um sistema imunológico mais suscetível para outras comorbidades, provocando maiores índices de óbitos. Esse desfecho foi encontrado por meio desta revisão, pois evidenciou que o risco de mortalidade por TB entre pacientes tabagistas é 2,6 vezes maior do que entre os não-fumantes[21,33], bem como trouxe uma associação da TB e o consumo ativo de tabaco com os óbitos[34].

Para proporcionar um maior controle e eliminação da TB, se faz necessário o desenvolvimento de estratégias que busquem reduzir ou cessar o tabagismo entre a população geral, visto que o hábito de consumir tabaco expõe o indivíduo a maiores riscos de infecções pulmonares. Portanto, é relevante enfatizar o controle do tabagismo em países que apresentam uma alta incidência de casos de TB, bem como buscar estratégias de triagem e rastreamento de TB entre os tabagistas, visto que esses indivíduos são uma população de risco, apresentando maiores chances do adoecimento por TB. 


\section{CONCLUSÃO}

Nesse contexto, evidencia-se um grave problema de saúde pública a nível mundial, principalmente em países onde a taxa de tabagismo é elevada. Assim, este estudo mostrou uma forte associação entre o consumo do tabaco e o aumento nas chances de desfechos desfavoráveis do tratamento da TB, como faIha/abandono do tratamento e óbitos.

Portanto, se faz necessário o desenvolvimento de estratégias de prevenção e promoção na atenção básica, visando uma redução/cessação no consumo de tabaco entre a população geral, já que quando o indivíduo possui o hábito de fumar encontra-se vulnerável para o desenvolvimento da TB. Além disso, nos pacientes diagnosticados, é fundamental a implementação de ações de redução de danos, bem como a cessação do tabaco, por meio de consultas terapêuticas e escuta ativa na estratégia de saúde da família.

\section{REFERÊNCIAS}

1. World Health Organization. Global tuberculosis report 2017 [Internet]. Geneva: World Health Organization; 2017. [cited 2019 Aug 25]. Available from: https://www.who.int/tb/ publications/global_report/gtbr2017_main_ text.pdf?u\%20a=1

2. Khalakdina A, Pai M, Chang L, Lessa F, Smith KR. The risk of tuberculosis from exposure to tobacco smoke: a systematic review and meta-analysis. Arch Intern Med. 2007Feb; 167(4):335-42. doi: 10.1001/archinte.167.4.335.

3. Lin HH, Ezzati M, Murray M. Tobacco smoke, indoor air pollution and tuberculosis: a systematic review and meta-analysis. PLoS Med. 2007Jan; 4(1):20. doi: 10.1371/journal. pmed.0040020.

4. Van ZylSmit RN, Pai M, Yew WW, Leung CC, Zumla A, Bateman ED, et al. Global lung health: the colliding epidemics of tuberculosis, tobacco smoking, HIV and COPD. Eur Clin Respi J. 2010Jan; 35(1):27-33. doi: 10.1183/09031936.00072909.

5. Rabahi MF. Tuberculose e Tabagismo. Pulmão RJ. [Internet]. 2012 [citado 2019 jul. 20];21(1):469. Disponível em: http://www.sopterj.com.br/ wp-content/themes/_sopterj_redesign_2017/_ revista/2012/n_01/11.pdf

6. Basu S, Stuckler D, Bitton A, Glantz SA. Projected effects of tobacco smoking on worldwide tuberculosis control:mathematical modeling analysis. BMJ. 20110ct; 343:d5506 doi: 10.1136/bmj.d550.

7. Instituto Brasileiro de Geografia e Estatística (IBGE). Pesquisa Nacional de Saúde 2013: percepção do estado de saúde, estilos de vida 
e doenças crônicas, Brasil. [Internet]. Rio de Janeiro: IBGE; 2014. [citado 2019 ago. 12]. Disponível em: http://biblioteca.ibge.gov.br/ visualizacao/livros/liv91110.pdf.

8. Novotny T, Hendrickson E, Soares ECC, Sereno A, Kiene SM. HIV/AIDS, tuberculosis, and tobacco in Brazil: a syndemic that calls for integrated interventions. Cad Saúde Pública. 2017Sep; 33(Suppl 3):e00124215. doi: 10.1590/0102311X00124215.

9. World Health Organization. International Union against Tuberculosis and Lung Disease. The Union monograph on TB and tobacco control: joining efforts to control two related global epidemics. [Internet]. Geneva: WHO; 2007. [cited 2019 Aug 10]. Available from: https:// apps.who.int/iris/handle/10665/43812

10. Moher D, Liberati A, Tetzlaff J, Altman DG, PRISMA Group. Preferred reporting items for systematic reviews and metaanalyses: The PRISMA Statement. PLoS Med. 2009Jul; 6(7):e1000097. doi: 10.1371/journal.pmed.1000097.

11. Balakrishnan S, Rakesh PS, Viswanathan K, Nelson V, Simon S, Nair SA. Higher risk of developing active TB among adult diabetics exposed to TB during childhood: A study from Kerala, India. J Family Med Prim Care. 2019Feb; 8(2):695-700. doi: 10.4103/jfmpc. jfmpc_344_18

12. Nandasena S, Senavirathna C, Munasinghe C, Wijesena C, Sucharitharathna R. Characteristics and sputum conversion of tuberculosis (TB) patients in Kalutara, Sri Lanka. Indian J Tuberc. 2019Jan; 66(1):76-80. doi: 10.1016/j. ijtb.2018.04.008.

13. Oliveira SM, Altmayer S, Zanon M, Sidney-Filho LA, Moreira ALS, Tarso PT, et al. Predictors of noncompliance to pulmonary tuberculosis treatment: an insight from South America. PLoS Med. 2018Sep; 13(9):e0202593. doi:10.1371/ journal.pone.0202593.

14. Tahseen S, Shahnawaz H, Riaz U, Khanzada FM, Hussain A, Aslam W. Systematic case finding for tuberculosis in HIV-infected people who inject drugs: experience from Pakistan. Int J Tuberc Lung Dis. 2018Feb;22(2):187-93. doi: 10.5588/ijtld.17.0390.

15. Mwiru RS, Nagu TJ, Kaduri P, Mugusi F, Fawzi W. Prevalence and patterns of cigarette smoking among patients co-infected with human immunodeficiency virus and tuberculosis in Tanzania. Drug Alcohol Depend. 2017Jan;170:128-32. doi: 10.1016/j. drugalcdep.2016.11.006.

16. Lindsay RP, Shin SS, Garfein RS, Rusch ML, Novotny TE. The association between active and passive smoking and latent tuberculosis infection in adults and children in the united states: results from NHANES. PLoS One. 2014Mar; 9(3):e93137. doi: 10.1371/journal. pone.0093137.

17. Alavi-Naini R, Sharifi-Mood $B$, Metanat $M$. Association between tuberculosis and smoking. Int J High Risk Behav Addict. 2012; 1(2):71-4. doi: 10.5812/ijhrba.5215.

18. Gillani S, Sulaiman SAS, Ali JA. Clinical modalities and therapeutic outcomes; between eversmokers versus never-smokers of tuberculosis patients in Penang, Malaysia. Eur J Gen Pract. 2010Jan; 7(4):389-97. doi.org/10.29333/ ejgm/82891.

19. Lindoso AABP, Waldman EA, Komatsu NK, Figueiredo SM, Taniguchi M, Rodrigues LC. Profile of tuberculosis patients progressing to death, city of São Paulo, Brazil, 2002. Rev Saúde Pública. 20080ct; 42(5):805-12. doi. org/10.1590/S0034-89102008000500004. 
20. Watkins RE, Plant AJ. Does smoking explain sex differences in the global tuberculosis epidemic? Epidemiol Infect. 2006Apr; 134(2):333-9. doi: $10.1017 /$ S0950268805005042.

21. Gajalakshmi V, Peto R, Kanaka TS, Jha P. Smoking and mortality from tuberculosis and other diseases in India: retrospective study of 43000 adult male deaths and 35000 controls. Lancet. 2003Aug; 362(9383):507-15. doi: 10.1016/S0140-6736(03)14109-8.

22. Hemanth-Kumar AK, Kannan T, Chandrasekaran V, Sudha V, Vijayakumar A, Ramesh K, et al. Pharmacokinetics of thrice-weekly rifampicin, isoniazid and pyrazinamide in adult tuberculosis patients in India. Int J Tuberc Lung Dis. 2016Sep; 20(9):1236-41. doi: 10.5588/ijtld.16.0048.

23. Baghaei $P$, Esmaeili $S$, Farshidpour M, Javanmard P, Marjani M, Moniri A, Nemati K. Diagnosing active and latent tuberculosis among Iranian HIV-infected patients. Clin Respir J. 2018Jan; 12(1):62-7. doi: 10.1111/crj.12479.

24. Ephrem T, Mengiste B, Mesfin F, Godana W. Determinants of active pulmonary tuberculosis in Ambo Hospital, West Ethiopia. Afr J Prim Health Care Fam Med. 2015Sep; 7(1):a608. doi.org/10.4102/phcfm.v7i1.608.

25. Gegia M, Magee MJ, Kempker RR, Kalandadze I, Chakhaia T, Golub JE, et al. Tobacco smoking and tuberculosis treatment outcomes: a prospective cohort study in Georgia. Bull World Health Organ. 2015Jun;93(6):390-9. doi: 10.2471/BLT.14.147439.

26. Kirenga BJ, Ssengooba W, Muwonge C, Nakiyingi L, Kyaligonza S, Kasozi S, et al. Tuberculosis risk factors among TB patients in Kampala, Uganda: implications for tuberculosis control. BMC Public Health. 2015Jan; 15(1):13. doi: 10.1186/ s12889-015-1376-3.
27. Ajagbe OB, Kabair Z, O'Cnnor T. Survival analysis of adult tuberculosis disease. PLoS One. 2014Nov; 9(11):e112838. doi.org/10.1371/ journal.pone.0112838.

28. Magee $M J$, Kempker RR, Kipiani M, Tukvadze $N$, Howards PP, Narayan KM, et al. Diabetes mellitus, smoking status, and rate of sputum culture conversion in patients with multidrug-resistant tuberculosis: a cohort study from the country of Georgia. PLoS One. 2014Apr; 9(4):e94890. doi: 10.1371/journal.pone.0094890.

29. Naidoo P, Peltzer K, Louw J, Matseke G, McHunu G, Tutshana B. Predictors of tuberculosis (TB) and antiretroviral (ARV) medication nonadherence in public primary care patients in South Africa: a cross sectional study. BMC Public Health. 2013Apr; 13:396. doi: 10.1186/14712458-13-396.

30. Singh PN, Yel D, Kheam T, Hurd G, Job JS. Cigarette smoking and tuberculosis in Cambodia: findings from a national sample. Tob Induc Dis. 2013Mar; 11(1):8. doi: 10.1186/1617-962511-8.

31. Slama K, Tachfouti N, Obtel M, Nejjari C. Factors associated with treatment default by tuberculosis patients in Fez, Morocco. East Mediterr Health J. [Internet]. 2013 [cited 2019 Aug 30]; 19(8):687-93. Disponível em: https:// apps.who.int/iris/handle/10665/118526.

32. Dujaili JA, Sulaiman SAS, Awaisu A, Muttalif AR, Blebil AQ. Outcomes of tuberculosis treatment: a retrospective cohort analysis of smoking versus non-smoking patients in Penang, Malaysia. J Public Health. 2011Apr;19(2):18389. doi:10.1007/s10389-010-0365-3.

33. Pednekar MS, Gupta PC. Prospective study of smoking and tuberculosis in India. Prev Med. 2007Jun; 44(6):496-8. doi: 10.1016/j. ypmed.2007.02.017. 
34. Maruza M, Alburquerque MFPM, Coimbra I, Moura LV, Montarroyos UR, Miranda-Filho DB, et al. Risk factors for default from TB treatment in HIV-infected individuals in the state of Pernambuco, Brazil: a prospective cohort study. BMC Infect Dis. 2011Jan;11(1):351. doi 10.1186/1471-2334-11-351.

35. Laniado-Laborín R. Smoking and chronic obstructive pulmonary disease (COPD). Parallel epidemics of the 21 century. Int J Environ Res Public Health. 2009Jan; 6(1):209-24. doi: 10.3390/ijerph6010209.

36. Barboza ML, Barbosa ACB, Spina GD, Sperandio EF, Arantes RL, Gagliardi ART, et al. Association between physical activity in daily life and pulmonary function in adult smokers. J Bras Pneumol. 2016Mar-Apr; 42(2):130-5. doi. org/10.1590/S1806-37562015000000102.

37. Silva DR, Muñoz-Torrico M, Duarte R, Galvão T, Bonini $\mathrm{EH}$, Arbex FF, et al. Fatores de risco para tuberculose: diabetes, tabagismo, álcool e uso de outras drogas. J Bras Pneumol. 2018mar; 44(2):145-52. doi. org/10.1590/s1806-37562017000000443.

38. Sá AMM, Santiago LA, Santos NV, Monteiro NP, Pinto PHA, Lima AM, et al. Causas de abandono do tratamento entre portadores de TB. Rev Soc Bras Clin Med. [Internet]. 2017 [citado 2019 jun. 20]; 15(3):155-60. Disponível em: http://pesquisa.bvsalud.org/portal/resource/ pt/biblio-875434.

39. Campani STA, Moreira JS, Tietbohel CN. Pulmonary tuberculosis treatment regimen recommended by the Brazilian National Ministry of Health: predictors of treatment noncompliance in the city of Porto Alegre, Brazil. J Bras Pneumol. 2011Dec; 37(6):776-82. doi. org/10.1590/S1806-37132011000600011.

40. 40. Santos TA, Martins MMF. Perfil dos casos de reingresso após abandono do tratamento da tuberculose em Salvador, Bahia, Brasil. Cad Saúde Colet. 2018;26(3): 233-40. doi. org/10.1590/1414-462×201800030235.

41. Yang JJ, Yu D, Wen W, Shu XO, Saito E, Rahman $\mathrm{S}$, et al. Tobacco smoking and mortality in Asia: a pooled meta-analysis. JAMA Netw Open. 2019Mar; 2(3):e191474. doi: 10.1001/ jamanetworkopen.2019.1474.

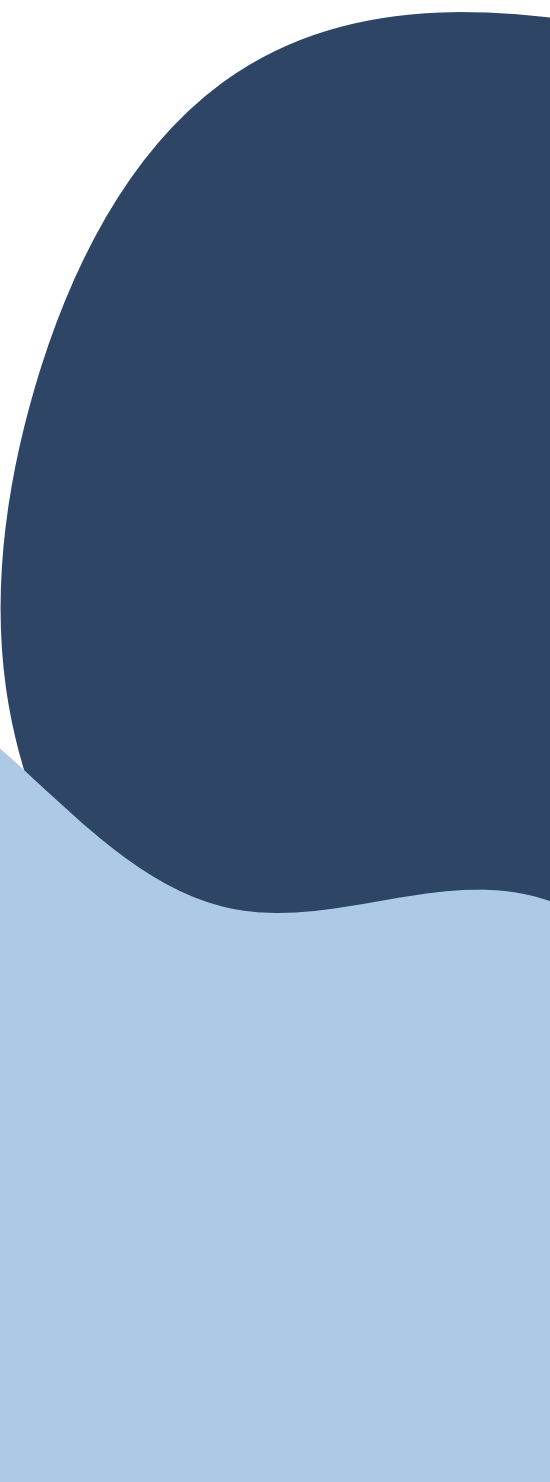

